\title{
Modelling the webspace of an intranet
}

\author{
Roelof van Zwol and Peter M.G. Apers \\ University of Twente, Enschede, the Netherlands \\ \{zwol, apers\}@cs.utwente.nl
}

\begin{abstract}
Searching the internet using the currently available search engines is not satisfactory. The techniques used there focus on the extraction of relevant information directly from the documents available on the web. We introduce a new approach, which aims at describing the content of a webspace, formed by a collection of related documents, instead of looking at the single documents. By identifying concepts and the relationships among them, the content of a webspace is described semantically in a schema for the webspace. The main objective is that by following this approach we can start querying the content of a collection of related documents rather than the content of a single document.

In this paper we introduce a model for webspaces that allows us to describe the concepts at a semantical level, in terms of classes, associations over classes, and attributes of classes. At the syntactical level we use XML, to describe information as instantiations of the concepts defined in the webspace schema. Dealing with data on the web, implies dealing with semi-structured data. We discuss how this relates to our model for a webspace and show how to deal with these aspects efficiently when going towards an implementation.

keywords: webspace-model, semi-structured, XML, concept-based search, Moa object algebra, organizing webspace.
\end{abstract}

\section{Introduction}

Large collections of electronic data are made available via the web. Most of the time the format of this data does not apply to a rigid structure. And therefore cannot be stored easily using the existing relational or object-oriented models $[15,6]$. When modelling data for the web, the focus used to be more on the presentation of data than on the content aspect. But with the rise of XML, structural aspects of data stored in documents on the web can be made explicit. The next step is to exploit these structural aspects, when searching for information on the web.
Currently, there are two ways of querying the web. One can search by association or by convergence. Searching by association is typically done using search engines. As a result, one gets stuck with huge lists of possibly relevant documents. Basically this comes down to lack of power in query formulation. Query languages $[18,23]$ developed to query XML documents are far more expressive, but rather focus on querying single XML documents, instead of a wide variety of documents. Searching by convergence is done when one searches for information, from a certain start point and then simply browses through all the linked documents. More advanced techniques are based on ontologies, where an information space is built up enabling users to walk through the webspace of an intra-net [20].

In the webspace model, as proposed here, we combine these two approaches for querying document collections. Such a collection should only contain documents that are related to a specific domain. These collections can typically be found when looking at an intranet environment. Instead of aiming on querying information stored in a single document, we intend to query the content of a webspace, which contains a collection of related documents. This approach has the mayor advantage that we can formulate queries that combine information stored in several documents. The purpose of the webspace model is to provide a semantical level of abstraction where, in terms of concepts and relationships among them, the content of a webspace is described.

Once such a schema for a webspace is defined, users can make instantiations of these concepts by marking up their information in XML documents. The tagging used in these $\mathrm{XML}$ documents refers to the concepts defined in the webspace schema.

It is not our intention to store entire documents in the database. But only data describing the concepts used in the XML documents will be stored, following a meta database approach. Combined with the framework for multimedia information retrieval, as described in [21], this enables us to build a search engine that supports both content- and concept-based query formulation. The concept-based part allows us to formulate queries over concepts used in different XML documents, i.e. the objects (or data) stored in 
different XML documents are instantiations of concepts defined in the webspace schema. We will illustrate this with some sample queries. Extending this with a content-based information retrieval framework will make our approach even more powerful, since this allows querying the content of text-fragments and other available multimedia objects as well.

The focus in this article will be on modelling and query formulation issues for concept-based retrieval. We will discuss this by presenting our ideas for modelling webspaces and show how a webspace schema can be used to formulate queries that integrate information described in several documents. Next we will discuss the semi-structured aspects of our model. By formalizing the webspace model three forms of semi-structuredness can be identified. This requires a flexible data model that is capable of storing the data efficiently. From a database point of view we will discuss the implementation of the architecture that we have set up. This is done in the light of the semi-structured aspects derived from the webspace model.

\section{State of the art}

The research done on modelling and querying semistructured data diverses in many directions, trying to cover the entire problem. A lot of research has focused on defining new data models and query languages for semistructured data (WebOQL [4], Lorel [1], STORED [7], $\mathrm{XQL}$ [18]), data integration issues (Tsimmis [16] and website management (Strudel [10] and Araneus [15]). These approaches work if the content in which documents are defined is known. In more general situations, additional information is required to describe the semantical meaning of concepts defined in a document. The Araneus project uses a relational approach to store the data that is presented on the web in a DBMS. We do not intend to store all the data in a database, but only the meta-data that can be derived. For dealing with the semi-structured aspects efficiently, we use an object oriented approach. The jCentral[12] search engine uses an RDF-based approach, using meta data that is available in java-based documents. One can specifically search for java applets, beans, or newsgroup articles, using this search engine. The need to describe the semantics of a document is also an issue in the On2Broker-project [9]. The need for abstraction is also expressed in [3] where a level of abstraction is introduced for a textual database. The difference with our approach is that we use a meta schema to model the webspace consisting of a collection of documents, instead of describing the structure of the documents available on that webspace. To gain a good overview: a comparison of several approaches, including our approach for storing semi-structured data, is done in [11]. For those interested in integration issues of multimedia information retrieval and DBMS we refer to [21], where those matters are discussed.

\section{Organization of this paper}

In the remainder of this paper we discuss modelling aspects of semi-structured data for the web in the light of our model for a webspace (Section 2). To illustrate the power of using schemas for describing the content of a webspace some queries are evaluated in Section 2.5. The formalization of the webspace model is discussed in Section 3. The database side is discussed in Section 4 where we give a short introduction of the principles behind Moa, our object algebra, and we discuss the consequences of the semi-structured aspects of the webspace model at implementation level. Finally, we will come to our conclusions and discuss future work in Sections 5 and 5.1.

\section{Modelling data on the intranet}

Due to the irregular structure of data on the web it is hard to model such data using the traditional data models of existing databases. Following an object-oriented approach, the same objects do not necessarily have all the attributes present. The same attribute might even have different types in different objects. To make it even worse, semantically related information can be represented differently in different objects. Data that inhabit these characteristics is called semi-structured data [19].

\subsection{Describing the content of a webspace in a schema}

Instead of building up index structures directly from the documents we aim to model a webspace, which contains a collection of related documents. The main objective is that by following this approach we can start querying the content of a collection of documents rather than the content of a single document. In Section 2.5 we will illustrate this with some sample queries.

The purpose of the webspace model is to provide a semantical level of abstraction where, in terms of concepts and relationships among them, the content of a webspace is described. We base our model on the class diagramming technique of the Unified Modelling Language (UML) [8]. UML provides a framework with design techniques for object-oriented modelling. Concepts available are defined in the webspace schema by means of classes, associations between classes, attributes of classes, and synonyms for classes. Instantiations of the concepts defined can then be described by the tags in XML documents.

With the XML standard [22] authors are no longer limited 
to the prescribed set of tags defined in HTML to mark-up their documents. In XML authors are free to define new tags to describe the content of their documents more accurately. This makes XML a very good vehicle for describing concepts at the syntactical level.

Besides the problem that XML data is not commonly available at the moment, applying this approach to the entire World-Wide Web would be too ambitious. The topics available on the web are too diverse, to be described in a single webspace. Therefore, we focus at the webspace of an intranet, which provides an environment where the documents are likely to be dealing with related content.

\subsection{Example: the Lonely Planet webspace}

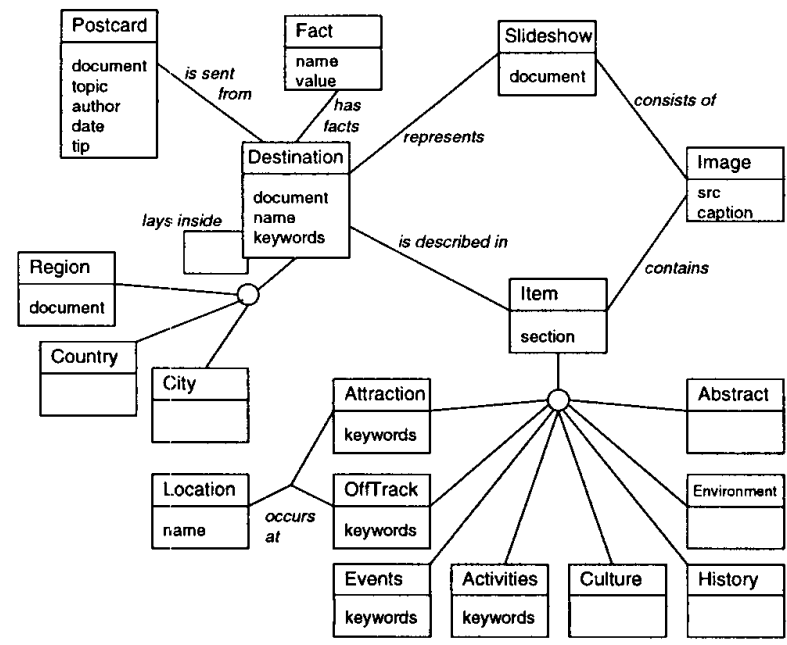

Figure 1. Schema for the Lonely Planet webspace

To be more concrete, we will illustrate by an example how concepts defined for a webspace can result in a schema for that webspace and how instantiations of classes can be defined in XML documents. Therefore we base our example on the web site of the Lonely Planet (http://www.lonelyplanet.com). This web site contains a large collection of documents describing information about destinations, documents with slide-shows containing pictures of a destination, and documents describing a region with countries and cities laying inside that region. Finally a large collection of letters is available, describing specific information that is sent in by travelers from a certain destination. Figure 1 describes a webspace schema that is set up, based on the concepts that were identified for the different documents of Lonely Planet web site. By integrating those concepts in a schema the content of the documents of this webspace is described at a semantical

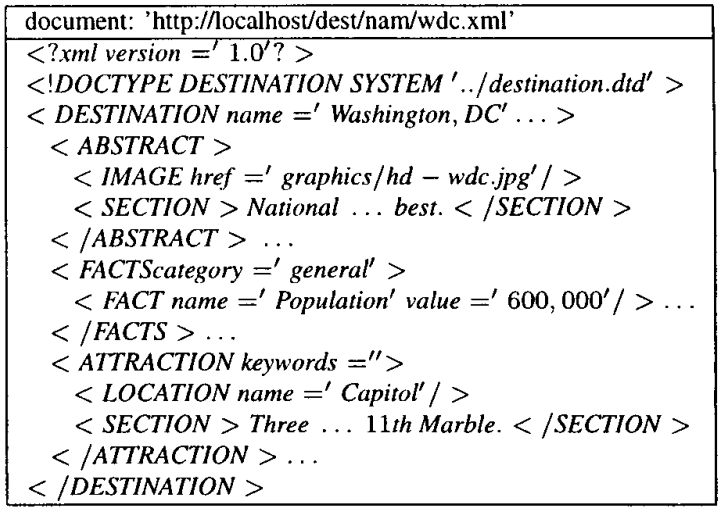

Table 1. XML fragment of the destination "Washington, DC"

level.

In Table 1 a fragment of an XML document is given. It describes an instantiation of the class Destination. By using a mapping mechanism as described in Section 2.4, the object describing the destination can easily be reconstructed. For this reconstruction it is required that all the elements and attributes used in the XML documents are defined in the webspace schema.

\subsection{Extensibility of the webspace model}

We already expressed the need of a flexible data model. Besides that the model should also have good extensibility properties, since information on the web is likely to change over time. When new data becomes available, which can not be properly defined in the current webspace schema, the schema should be extended at the semantical level and new tags should be defined for the XML documents at the syntactical level.

Extensibility at the semantical level requires that authors should be able to add new concepts to the webspace schema in term of classes, associations, and attributes. This of course may not be in conflict with the existing concept definitions, since this might result in semantically related information to be represented differently in different objects. A typical example of this is when in one document the concept car is used, while another document uses the concept automobile. Both concepts are semantically related, and are likely to have common attributes defined. Therefore we introduce synonyms, which allows authors to use synonym concepts for describing the data in their documents. Each new synonym concept refers to a single concept of the existing webspace schema.

The task of extending the webspace schema can be simpli- 
fied if authors refer to concepts that are defined in standards (Dublin Core [25] and RDF [24]) at the internet level. By integrating these standards larger domains of the internet can be covered.

\subsection{Mapping mechanism for deriving the meta data}

When not conforming to such standards, the information stored in the XML documents should be derived using a mapping mechanism. At this point it is not our intention to give a complete description on how to derive meta data from the XML documents. Several approaches are possible resulting in more flexible or smarter mapping mechanisms. XML documents have a tree-based structure. Starting from the root element the tree can be searched for objects, that form instantiations of classes defined in the webspace schema. A first and simple mapping can be realized using the following rules:

- The root element should always refer to a class definition of the webspace schema (the source of that document is also a document attribute within that class.

- If the instantiation of a class (or one of its synonyms) is found as an element of an XML document. (l) All the attributes of that element should be searched for corresponding attributes of that class. (2) All the child elements should be processed to find mappings of $(i)$ possible attributes belonging to this class, (ii) associations in which the class can participate or (iii) associated classes.

- If the element is defined as an attribute in the webspace schema, the data described in the element's children should be derived. The meta-data should be extracted from those childs and stored as an attribute of the object involved.

- If the element refers to an association of the webspace schema, then $(I)$ all the attributes of the element should be processed, as well as (2) all the element's children to find instances of classes participating in the association.

\section{Example}

Returning to the example of Section 2.2 the XML fragment of Table 1 can be mapped using the mapping mechanism. Starting from the root element of the XML document, one finds the tag $\langle$ DESTINATION ... $\rangle$, referring to the class destination of the webspace schema given in Figure 1. Parsing all the attribute and child elements, will result in the reconstruction of the destination-object.

\subsection{Querying the webspace}

Once a schema for the webspace is defined, users can start querying the webspace based on the concepts defined for that webspace. By using a graphical interface, a view on (a part of) the schema of a webspace is given. The user can browse through this view and select relevant concepts. By doing so he can adjust his view until all the desired concepts are selected. Then fill in all the attributes of his interest and automatically compose the object-oriented query. When a webspace grows larger, the size of the schema will grow larger. To prevent the view on the schema to become too detailed, the user should start with a view that contains only a subset of the entire webspace schema. By browsing through the schema a user can easily change his view to find other concepts. We will illustrate by an example what kind of queries can be formulated, using the webspace schema. It can be easily seen that these queries are far more powerful than when a traditional search engine is used.

\section{Example}

Taking a look at the example of the Lonely Planet again, a user might want to ask the following questions, while searching through the destination pages:

- Retrieve all Destination.documents, that have facts, with Fact.name "population" and Fact.value greater than 500.000 , laying inside a Region, with Region. name "Asia".

- Retrieve all Postcard.documents, that are sent from a destination, with Fact . name is "population" and Fact.value greater than 500.000 and where Postcard. tip contains "danger"

\begin{tabular}{|c|c|}
\hline $\begin{array}{l}\text { select d.document } \\
\text { from Region } r \text {, } \\
\text { d in r.inside } \\
\text { where r.name }=^{\prime} \text { Asia' and } \\
\text { exists } f \text { in d.facts }: \\
\quad \text { f.name }=^{\prime} \text { population' } \\
\text { and f.value }>500.000\end{array}$ & $\begin{array}{l}\text { select p.document } \\
\text { from Postcard p, } \\
\text { d in p.SendFrom } \\
\text { where (exists } f \text { in d facts : } \\
\text { f.name }=^{\prime} \text { population' and } \\
\text { f.value }>500.000) \text { and } \\
\text { p.tip } \sim *^{\prime} \text { danger' }\end{array}$ \\
\hline (a) & (b) \\
\hline
\end{tabular}

Table 2. Sample queries

In Table $2 a$, and 2.b, the OQL syntax for these queries is given. The first query, where the user is interested in destination documents is a typical example of a query that uses information stored in several XML documents. In this case it uses information from documents describing a destination for finding some facts defined for that destination, but also meta-data from documents describing a region is used. 
The second query, also uses information stored in different XML documents. But it differs from the first query in the fact that this query also uses content based search techniques, for doing some textual information retrieval on the content of Postcard. tip. This query gives a typical example of how concept based search and content based information retrieval can be combined using our approach for modelling a webspace.

\section{Formal definition of the webspace model}

By giving a formal definition of the webspace model, better insight in the semi-structured aspects of the model is gained. It also allows us to identify the constraints for specifying webspaces. For the formalization we use the $\mathrm{Z}$ specification language [17]. We will start with the definition of several concept spaces needed for the webspace model.

\subsection{Concept spaces}

Before we come to the formal definition of a webspace model, the notion of concepts has to be defined. Informally we consider a concept to have a semantical meaning, captured by a unique name. Since there is no need to further specify this for the formalization, a simple declaration of Concept is enough. The collection Concept webspace $_{\text {iden- }}$ tifies a finite set of Concept, containing only those concepts that are used within a certain webspace. We also introduce the collection Concept $_{\text {basicwebspace, }}$ as a subset of Concept $_{\text {webspace, }}$ which might be equal to Concept $t_{\text {webspace }}$ if no synonyms are defined for that webspace.

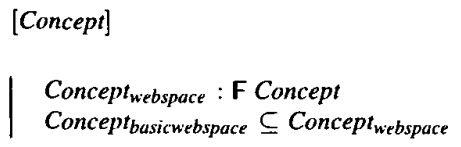

A webspace partition consists of the concept spaces: Concept $_{\text {class, }}$, Concept association, Concept $_{\text {attribute, }}$, and

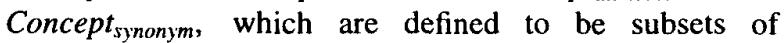
Concept $_{\text {webspace. It respectively represents the collection of }}$ concepts defined as classes, associations between classes, attributes of classes, and user-defined synonyms for classes. For the definition of our basic webspace model synonyms are left out. Therefore we define Concept basicwebspace $_{\text {as }}$ the conceptspace, formed by the partition of Concept $t_{\text {class }}$, Concept association $_{\text {and }}$ Concept $t_{\text {attribute. A concept belonging }}$ to Concept $_{\text {class }}$ can never be reused as an association or attribute in the same webspace.

By introducing synonyms, we need to extend our notion

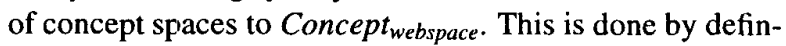
ing the partition over Concept $_{\text {basicwebspace }}$ and Concept synonym $_{\text {sy }}$ as Concept $t_{\text {webspace. }}$.

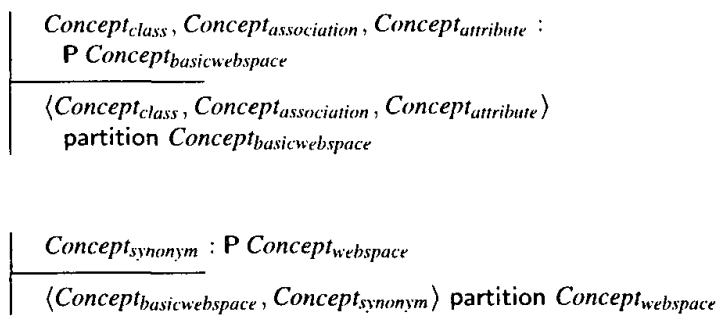

\subsection{The Webspace model}

We consider a webspace to be the space available on the web, containing a collection of related documents. A webspace model describes the webspace at a higher level of abstraction. It describes the concepts and the relationships among them. For our model of a webspace we use a graphbased approach, based on the class diagramming method of UML [8]. Therefore, we distinguish classes, associations, and attributes. We enrich our model with the notion of types for attributes. Although we make an explicit distinction between multimedia-based types and ordinary types at the level of implementation, there is no need to do so when defining the webspace model. From a user point of view all types should be "equal".

\section{[Type]}

\section{Class}

A Class is defined by its name and a set of attibutes defining the properties of that Class. The following constraints apply for a Class:

- The name of a Class is a member of Concept class. $_{\text {. }}$.

- The attributes defined for a Class forms a subset of Concept $t_{\text {atribute, }}$, identified by the names of the concepts used.

- An attribute, belonging to the set of attributes is only properly defined if there is a Type assigned. In its most basic form this will be the string representation, which can be immediately derived from the document. This combined with the previous requirement can be expressed as: Concept attribute $\rightarrow$ Type.

- Each attribute should have at least one type assigned $\left(\mathbb{F}_{1}\right.$ Type $)$.

This last constraint, where each attribute can have more than one type representing that attribute, expresses the first semi-structured aspect of our model. Data available on the 
web does not apply to strict typing rules. For example a date can be represented as a string (" 20 December 1999") or as an integer (122099). The second semi-structured aspect also follows from the Class definition. Objects belonging to the same class do not necessarily have all the attributes of a class as their properties available.

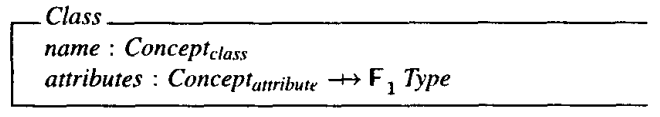

\section{Association}

An Association is defined by its name and a set of classes, called associates, defining the properties of that Association. The following constraints apply for an Association:

- The name of an Association is a member of Concept $t_{\text {association }}$

- Associates describes the set of Class, participating in an Association.

- There is at least one Class participating in an Association.

- $\mathrm{N}$-ary associations between classes are allowed.

\begin{tabular}{|l} 
Association \\
name $:$ Conceptassociation \\
associates $: \mathbf{P}$ Class \\
\hline associates $\neq \varnothing$ \\
\hline
\end{tabular}

\section{Webspaces}

The formal definition of athe webspace model is build up in four steps. We will start with describing the basic model, then extend this model, respectively, with the inheritance- , synonym- , and connected properties.

The Webspace basic consists of a collection of classes and a collection of associations. The following constraints apply for the definition of Webspace basic:

- All the concepts that belong to the set of Concept class will have to participate as a class in the webspace.

- The name of a Class is unique within a webspace.

- All the concepts that belong to the set of Concept $_{\text {association }}$ will have to participate as an association in the webspace.
- The name of an Association is unique within a webspace.

- the union of all attributes used in classes of the webspace should be equal to Concept $t_{\text {atribute }}$. Note that an attribute can be used to describe a property of more than one class and that the types of that attribute may differ over the different classes.

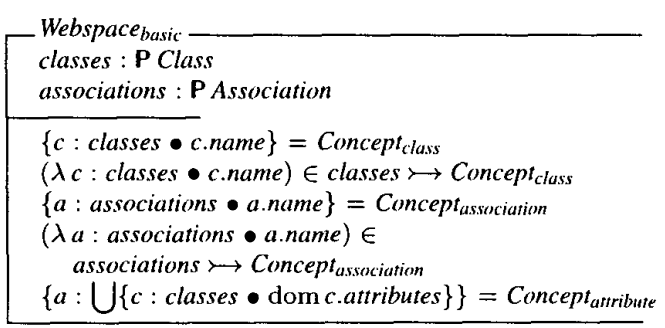

The first extension of the basic model to Webspace troduces the generalization of classes. To formalize this, the model is extended with generalizes, defined as a relation between classes of the webspace model. That a Class can never be a subclass of itself is expressed by adding the second constraint to the definition of Webspace ${ }_{\nu o}$.

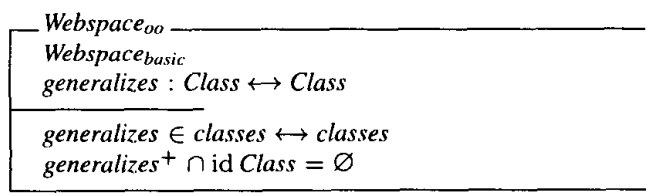

The second extension allows the use of synonyms, when defining a webspace schema. For each concept

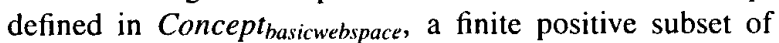
Concept $_{\text {synonym }}$ can be assigned. All the synonyms in Concept $t_{\text {synonym }}$ are defined, exactly once to a concept belonging to Concept $_{\text {basicwebspace. Introducing synonyms to the }}$ model, as discussed in Section 2.3, expresses the third semistructured aspect.

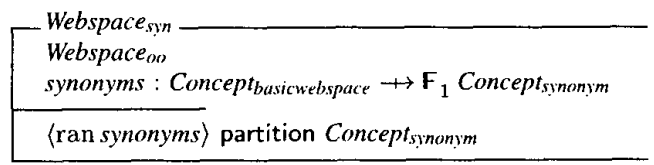

The final extension as defined in Webspace connected $_{\text {ex- }}$ presses that a webspace only consists of connected classes. So each class is connected to all other classes of the webspace by 1 or more intermediate associations.

From the formal specification of the webspace model, we have identified three forms of semi-structured aspects. 


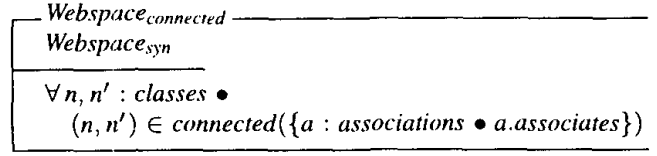

The first two forms, which occur at type and attribute level are dealt with in Moa, which forms the logical layer of our architecture, as discussed in Section 4.2. The third form, occurs at the class level, where synonyms are defined for classes. This form is directly dealt with at the conceptual layer of the architecture, which implements the webspace search engine (Section 4.1).

\section{Semi-structured aspects, the database side}

\subsection{Architecture}

The logical and physical layers of our architecture, as shown in Figure 2, are implemented by respectively Moa and Monet. The combination of Moa and Monet, as an object server, is already illustrated to be a powerful backend for several application domains, such as geographic information systems [26], multimedia information retrieval [21], and data mining [13]. On top of the architecture, given

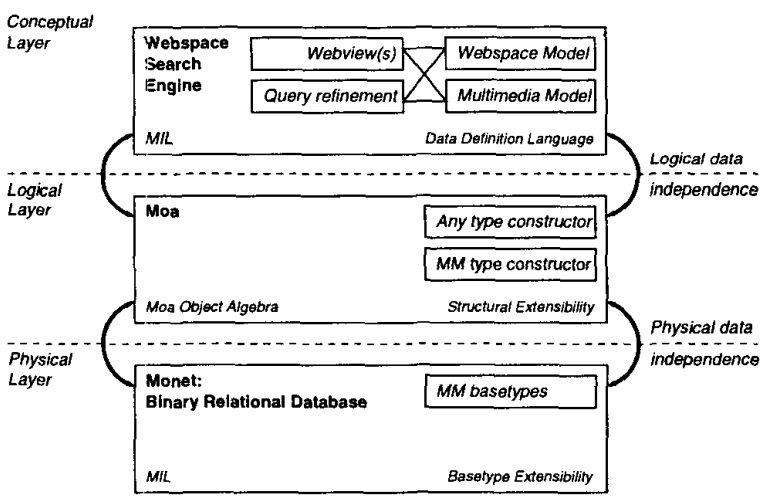

Figure 2. concept partitions of the extended webspace

in Figure 2, at the conceptual level we intend to provide OQL support for the query evaluation. The webspace model should be embedded in the data definition language of OQL, as well as the multimedia information retrieval framework as described by [21]. For the time being a direct translation of the webspace model onto the Moa data model will be used to realize a first implementation.

\section{The webspace search engine.}

This component is responsible for the query interface to the webspace schema. As can be seen from Figure 2 the search engine consists of four interacting components. Implementing the semi-structured aspects of the webspace model at the class level, it is also responsible for the mapping of synonym concepts to concepts defined in the webspace schema. Currently an OQL layer on top of Moa is being developed in our group, we aim at using OQL and its data definition language as intermediate between the graphical composition of queries using the webspace schema at the conceptual level and the Moa object algebra at the logical level. Integrating our approach with the framework for multimedia information retrieval requires a component responsible for query refinement and user interaction, as proposed in [21].

\section{Object server.}

At the physical layer of the object server we use Monet, an extensible parallel database kernel, based on a binary relational data model, which is developed at the University of Amsterdam and CWI since 1994. To provide data independence at the logical layer we use Moa [5]. Moa consists of a structural object-oriented data model and algebra.

\subsection{About Moa}

Since the efficient storage of semi-structured data depends heavily on Moa's structural extensibility a short summary of its data model and algebra is given here as an introduction. More detailed information on Moa and its design principles can be found in $[21,5]$. In Section 4.3 the implementation of the ANY-type constructor is explained. This extension is used to model the semi-structured aspects of the webspace model efficiently.

\section{Moa data model}

The principle of structural object-orientation forms the basis of Moa's data model. Moa assumes a finite set of ADT-style base types and structured types. The base types are implemented at the physical level of the object server. The internal structure of the types can only be accessed via the operations defined on those base types. Basically Moa's type system comes down to:

- base types: $\tau$ is a base type, if $\tau$ is an atomic type at the physical level.

- structured types: if $\tau_{1}, \cdots, \tau_{n}$, is a possible empty, list of types and $\mathcal{T}$ is a structure defined over $\tau_{1}, \cdots, \tau_{n}$, then $\mathcal{T}\left\langle\tau_{1}, \cdots, \tau_{n}\right\rangle$ is a structured type.

Structured types (type constructors) are defined at the logical level. They combine known types to create a 
structured type. In the basic MOA data model only the well-known structures SET, TUPLE, and $O B J E C T$ are defined. Extensions to the Moa data model, regarding the support for multimedia information retrieval can be found in [21]. To support the storage of semistructured data and the semi-structured aspects, as derived from the webspace model, a new type constructor was added to the Moa data model (4.3).

\section{Moa object algebra}

The Moa object algebra purely consists of the operations defined on the available atomic base types and its structural extensions. For example, on the SETstructure the (basic) operations select, project, join, nest, unnest, and others are defined. But also access to attributes of TUPLE's and to OBJECT is defined in the Moa framework. In the same manner methods that work on (atomic) base type can be invoked.

\subsection{ANY type constructor}

Several approaches $[2,1,7,11]$ are introduced to deal with semi-structured data. Modelling such data requires a flexible data model that supports irregular, implicit, and partial structure of data. To be able to deal with such data a new type structure is defined that allows data to be incomplete and irregular. In [27] we illustrated how the ANY type constructor can be used to integrate semi-structured data and structured data in the same data model. This integration provides us with the basis to query both structured and semistructured data in exactly the same manner.

Based on the definition of an identified value set, as given below, a formal specification of the ANY type constructor can be derived.

An identified value set $S$ is defined as a set of pairs $\left(i d_{i}, v_{i}\right)$ in which each value $v_{i}$ is associated with an identifier $i d_{i}$ that is unique within the value set:

$$
\left\{\left(i d_{i}, v_{i}\right) \mid i, j \in 1 \cdots n, i \neq j \rightarrow i d_{i} \neq i d_{j}\right\}
$$

Consider $I V S_{1}, \ldots, I V S_{n}$ as a collection of asynchronous identified value sets, the structure function $A N Y\left(I V S_{1}, \ldots, I V S_{n}\right)$ defines the new combined set:

$$
\left\{\left(i d_{i},\left\{v_{i j} \mid\left(i d_{i}, v_{i j}\right) \in I V S j\right\}\right) \mid\left(i d_{i} \in I V S_{1} \cup \ldots \cup I V S_{n}\right\}\right.
$$

There are two functions defined on the ANY structure, that allow the user to manipulate the ANY structure.

- The attr operation allows to select a specific attribute or a set of attributes from the ANY structure.

- The filled operation checks for the existence of objects, or for one of its attributes, belonging to the ANY structure.

\subsubsection{ANY and the semi-structured aspects of the web- space model}

The ANY structure is reused here to implement the semistructured aspects of the webspace model. The flexible structure properties of the ANY type constructor are needed to deal with semi-structured aspects at type and attribute level.

\section{Type level}

When looking at the webspace model at the type level, one can easily see that a certain attribute of a class might have different types assigned to it. In Moa several base types can be assigned to one attribute as is illustrated in Figure 4.b, where the date attribute can be stored either as a date base-type or as a string basetype. At this level the ANY functions as a collector structure for known base types, allowing instances to have multiple representations of the same attribute. In [14] similar problems like type coercion and overloading of types are discussed and classified as typical examples of ad-hoc polymorphism. In our approach operations defined on types involved can be invoked, when querying such a data structure.

\section{Attribute level}

At the attribute level, it is likely that instances of a certain class do not have all the attributes that are defined for that class, when dealing with semi-structured data. Normally, when modelling such problems in a object-oriented environment, introducing a new super class would be the solution. But since all the data is allowed to be incomplete or irregular, applying such an approach for semi-structured data would lead to very large and unmanageable schemas. Again the ANY type constructor is used (Figure 4.b), now as a collector structor for all known attributes of a class. Only existing attribute values are stored, as is shown in Figure 5, resulting in efficient storage at the physical layer.

\subsection{An example: From XML to Object server}

Now that the technical aspects of our system are discussed, we will show by an example how the 'meta'-data, necessary to fill the database can be derived from the XML documents. Given the webspace schema of the lonely planet example (Figure 1), XML documents describing postcards (Figure 3) will form instantiations of the class Postcard, it will also define the association to a Destination by its name. Figure 4.a shows the part of the webspace schema involved.

Although the XML documents both describe a postcard send from a destination, the postcard- objects will 


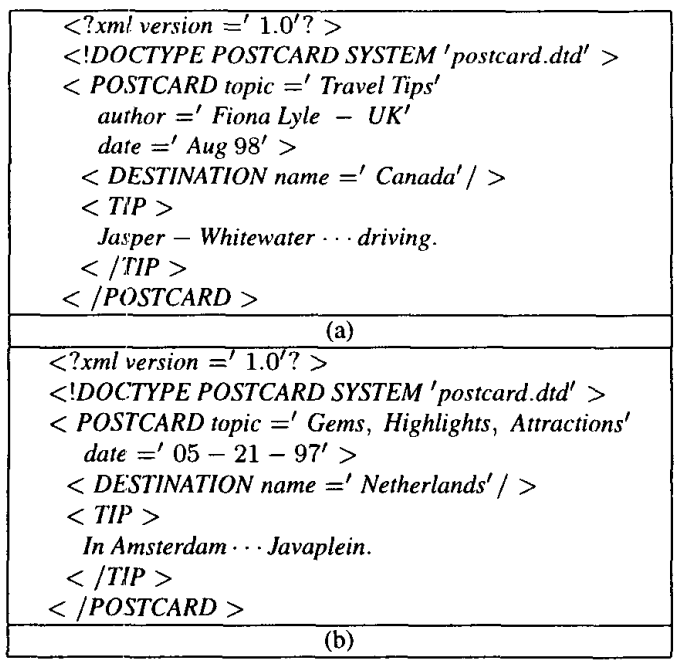

Figure 3. Postcard example: xml fragments.

have different properties defined. The postcard of Figure 3.b misses the author attribute. There is also a difference in the type of the date attribute. To deal with these types of semi-structured aspects the ANY type constructor is used as shown in Figure 5b. It shows the Moa data model of the classes postcard and destination. The outer ANY models the attributes, while the inner ANY is used to deal with type coercion.

Figure 5 shows the flattened representation of the semistructured class postcard at the physical layer. The binary tables only store the attribute values defined for the objects involved [27, 11].

\section{Conclusions}

By describing the content of a webspace, in terms of concepts and the relationships among them, we have gained a semantical level of abstraction. This allows us to do concept based search over a collection of related documents, as if they were modelled in an object oriented database schema. Using such a schema for a webspace enables us to formulate OQL-like queries. We can combine information stored in several documents and use it within a single query.

Dealing with data on the web (intranet), implies dealing with semi-structured data. Therefore we have introduced a webspace model. In this model concepts can be described that are defined for a webspace. This is done in terms of classes, associations over classes, attributes of classes, and synonyms for classes. By formalizing this model three forms of semi-structured aspects are identified. At type level, multiple types can be assigned to a single attribute (type coercion). At attribute level, instantiations of a class do not necessarily have the same or all attributes defined.

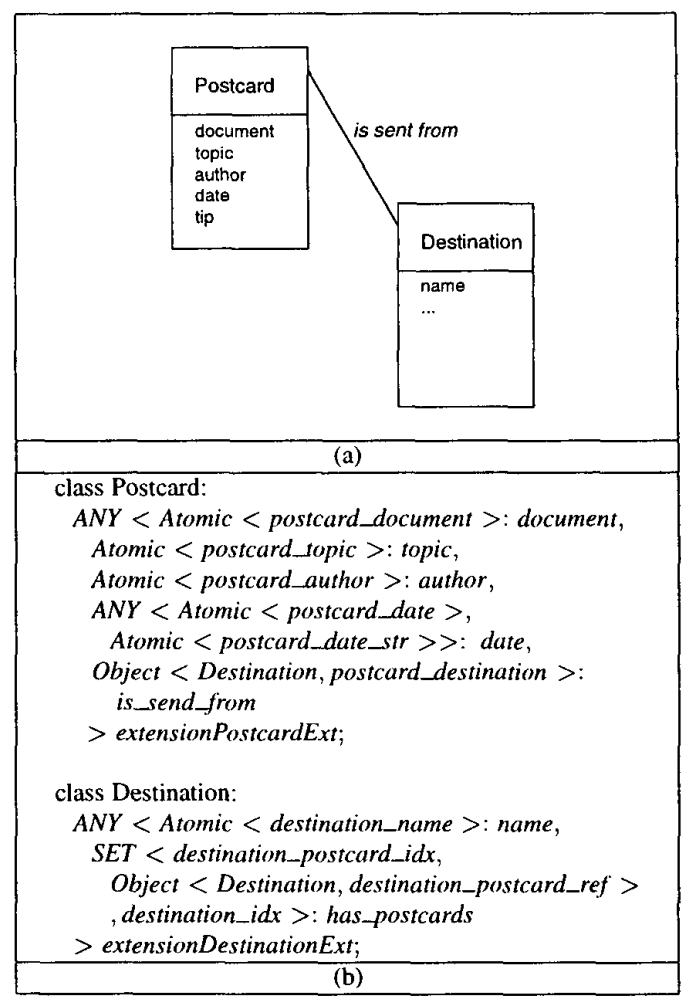

Figure 4. Postcard example: modelling issues.

Finally at class level, synonyms can be assigned to a class, since semantically related information on the web can be represented differently in different objects.

For the implementation of the webspace model, and its semi-structured aspects a three layer architecture is set up. We have shown how to map the webspace model onto the Moa data model, and how we can efficiently deal with the semi-structured aspects at attribute and type level, by using the ANY type constructor.

\subsection{Future work}

Integrating our work with the framework for multimedia information retrieval, will enable us to do both concept based and content based retrieval. On the other hand we want to concentrate on extending the search engine with an OQL-based query interface, as an intermediate layer between the webspace search engine and the Moa object algebra. When the size of the webspace grows, it is likely that the webspace schema will grow. To be able to find the concepts one searches for, we intend to use web-views, which visualizes a part of the webspace involved. By changing the web-view a user can identify all the concepts he is interested 


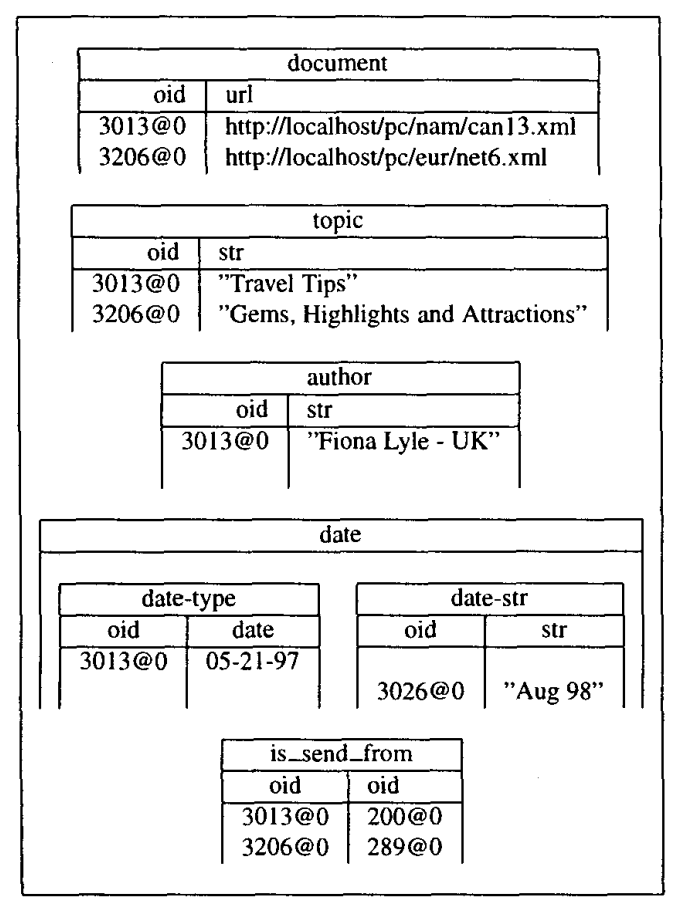

Figure 5. Postcard example: storage at physical layer.

in, before composing the query.

\section{References}

[1] D. Abiteboul, Quass, J. McHugh, J. Widom, and W. J. The lorel query language for semistructured data. journal of Dig. ital Libraries, pages 1(1):68-88, apr 1997.

[2] S. Abiteboul. Querying semi-structured data. In proceedings of the International Conference on Database Theory(ICDT97), Delphi, Greece, 1997.

[3] M. Agosti, G. Gradenigo, and P. Marchetti. A hypertext environment for interacting with large textual da tabases, 1992.

[4] G. Arocena and A. Mendelzon. WebOQL: Restructuring documents, databases and webs. In proceedings of Fourteenth IEEE International Conference on Data Engineering (ICDE98), 1998.

[5] P. A. Boncz, A. N. Wilschut, and M. L. Kersten. Flattening an Object Algebra to Provide Performance. In Proceedings of the IEEE International Conference on Data Engineering (ICDE), pages 568-577, Orlando, FL, USA, February 1998.

[6] V. Christophides, S. Abiteboul, S. Cluet, and M. Scholl. From structured documents to novel query facilities. In proceedings of SIGMOD94, 1994.

[7] A. Deutsch, M. Fernandez, and D. Suciu. Storing semistructured data with STORED. In proceedings of the ACM SIGMOD International Conference on Management of Data, 1999.
[8] D. D'Souza and A. Wills. Objects, Components, and Frameworks with UML. The Catalysis Approach. Addison-Wesley, 1998.

[9] D. Fensel, M. Erdmann, and R. Studer. On2broker: The very high idea. In proceedings of the 11th International Flairs Conference (FLAIRS-98), Sanibal Island, Florida, May 1998.

[10] M. Fernandez, D. Florescu, J. Kang, A. Levy, and D. Suciu. Catching the boat with Strudel: Experiences with a website management system. In proceedings of ACM SIGMOD Conference on Management of Data, Seattle, WA, 1997.

[11] D. Florescu and D. Kossmann. A performance evaluation of alternative mapping schemes for storing XML data in a relational database. Technical report, INRIA, Rocquencourt, May 1999.

[12] IBM. jcentral search engine. http://www.ibm.com/java/ jcentral/basic-search.html, 1999.

[13] M. Kersten, A. Siebes, M. Holsheimer, and F. Kwakkel. A data cube algebra engine for data mining. http://www.cwi. $\mathrm{nl} / \sim$ monet/papers/cube.ps.gz.

[14] C. L. and P. Wegner. On understanding types, data abstraction, and polymorphism, volume 17, pages 471-522. Association for Computing Machinery (ACM), Dec. 1985.

[15] G. Mecca, P. Merialdo, P. Atzeni, and V. Crescenzi. The Araneus guide to web-site development. Technical report, Dipartimento di Informatica e Automazione, Universita' di Roma Tre, Mar. 1999.

[16] Y. Papkonstantinou, H. Garcia-Molina, and J. Widom. Object exchange across heterogeneous information sources. In IEEE International Conference on Data Engineering, pages $251-260,1995$.

[17] B. Potter, J. Sinclair, and D. Till. An Introduction to Formal Specification and $Z$. Prentice Hall International Series in Computer Science. Prentice Hall, 1991.

[18] J. Robie, J. Lapp, and D. Schach. XML query language. http://www.w3.org/TandS/QLQL98/pp/xql.html, 1998.

[19] D. Suciu. Overview of semi structured data, pages 28-38. Number 4. SIGACT News, Dec. 1998.

[20] W. Veling. Aqua browser - the answers. online demo: http: //www.medialab.nl/, 1997.

[21] A. d. Vries. Content and multimedia database management systems. PhD thesis, University of Twente, Enschede, The Netherlands, Dec. 1999.

[22] W3C. Extensible markup language (XML). Technical report, World Wide Web Consortium (W3C), Feb. 1998.

[23] W3C. Q1'98 - the query languages workshop. http://www. w3.org/TandS/QL/QL98/, 1998.

[24] W3C. Resource description framework (RDF). Technical report, World Wide Web Consortium (W3C), Feb. 1999.

[25] S. Weibel, J. Kunze, C. Lagoze, and M. Wolf. Dublin Core metadata for resource discovery. The Internet Society, Sept. 1998.

[26] A. Wilschut, R. v. Zwol, and J. Flokstra. Road collapse in magnum. In proceedings of 6th International Symposuim on Advances in Geographic Information Systems, Nov. 1998.

[27] R. v. Zwol, P. Apers, and A. Wilschut. Modelling and querying semistructured data with Moa. In proceedings of Workshop on Query Processing for Semistructured Data and Non-standard Data Formats, Jerusalem, Israel, Jan. 1999. 